\title{
THE TIGHTNESS OF CERTAIN ALMOST COMPLEX SUBMANIFOLDS
}

\author{
CRISTIÁN U. SÁNCHEZ
}

(Communicated by Jonathan M. Rosenberg)

\begin{abstract}
This paper contains a proof of the following fact. If a map $f$ from a connected Riemannian manifold with an almost complex structure into a euclidean space has the following properties: (a) $f$ is nondegenerate and (b) for almost all height functions, the linear Hessian of the composition with $f$ commutes with the almost complex structure, then $f$ is tight. This gives some information about the homology groups of the manifold. This result yields a new proof of a well-known theorem of R. Bott.
\end{abstract}

\section{INTRODUCTION}

This paper deals with tight smooth maps of a compact Riemannian manifold into a Euclidean space. Let $M^{n}$ be a compact $n$-manifold, and let $f: M^{n} \rightarrow$ $\mathbf{R}^{N}$ be a $C^{\infty}$ map. For each unit vector $v \in S^{N-1}$ we may consider its "height function" $h_{v}(x)=\langle v, x\rangle \forall x \in \mathbf{R}^{N}$. As in [5] we say that $f$ above is nondegenerate if, for almost all vectors $v \in S^{N-1}$ (i.e., for the complement of a set of Lebesgue measure zero), the function $\varphi_{v}=\left(h_{v} \circ f\right)$ is a Morse function on $M$.

For a Morse function $\varphi: M \rightarrow \mathbf{R}$, we denote by $\mu_{k}(\varphi)$ the number of critical points of index $k$ and define $\mu(\varphi)=\sum_{k} \mu_{k}(\varphi)$. Let $F$ be a field and denote by $\beta_{i}(M, F)$ the $i$ th Betti number of $M$ with coefficients in $F$. Let $\beta(M, F)=$ $\sum_{i} \beta_{i}(M, F)$. The smooth function $f: M^{n} \rightarrow \mathbf{R}^{N}$ is called tight [5] in case $f$ is nondegenerate and, for any $v \in S^{N-1}$ for which $\varphi_{v}$ is a Morse function, it satisfies $\mu\left(\varphi_{v}\right)=\beta(M, F)$. For the functions $\varphi_{v}$ which are Morse, we have at a critical point $p$ the Hessian of $\varphi_{v} H\left(\varphi_{v}\right)$, which is a nondegenerate symmetric bilinear form which can be realized by a symmetric linear operator on $T_{p}(M)$,

Received by the editors April 6, 1989 and, in revised form, November 14, 1989.

1980 Mathematics Subject Classification (1985 Revision). Primary 53C15; Secondary 53C30, $53 \mathrm{C} 40$.

Key words and phrases. Almost complex structure, nondegenerate function, tight function, Hessian.

This research was partially supported by a grant from the Consejo de Investigaciones Cientificas y Tecnológicas de la Provincia de Córdoba (CONICOR), Argentina. 


$$
H\left(\varphi_{v}\right)(X, Y)=\left\langle A_{v} X, Y\right\rangle \quad \forall X, Y \in T_{p}(M) .
$$

We call $A_{v}$ the linear Hessian at $p$.

Remark. In connection with this notion of tightness see [6, Problem 1].

The objective of the present paper is to prove

(1.1) Theorem. Let $(M,\langle\rangle$,$) be a compact connected Riemannian mani-$ fold with an almost complex structure $J$. Assume that there is a $C^{\infty}$ function $f: M^{n} \rightarrow \mathbf{R}^{N}$ which satisfies the following conditions:

(i) $f$ is nondegenerate;

(ii) For each $\varphi_{v}=h_{v} \circ f$ which is a Morse function and each critical point $p$ of $\varphi_{v}$, the linear Hessian $A_{v}$ at $p$ satisfies

$$
A_{v} J_{p} X=J_{p} A_{v} X \quad \forall X \in T_{p}(M) .
$$

Then the function $f$ is tight and

(a) The integral homology of $M$ is free of torsion;

(b) The odd Betti numbers of $M$ vanish.

As we shall see this result generalizes Theorem A of M. R. Bott [2] and also Corollary 2 of T. Frankel [3, p. 6] which is already a generalization of Bott's result. The statement of Theorem $A$ in [2] is

(1.2) Theorem (M. R. Bott). If $G$ is a simply connected compact Lie group, $T$ is a torus of $G(\operatorname{dim} T \leq \operatorname{rank} G)$, and if $C(T)$ denotes the centralizer of $T$ in $G$ then the space $G / C(T)$ has the following properties:

(a) Its homology is free of torsion.

(b) Its odd Betti numbers vanish.

In the next section we make the necessary remarks to get (1.2) as a corollary to (1.1) as well as some comments about the connections of (1.1) with the results of T. Frankel [3] and S. Kobayashi [4]. $\S 3$ contains the very simple proof of (1.1).

In [3] T. Frankel studies 1-parameter groups of isometries on a compact Kähler manifold $(M, g, J)$. His main result is an equality relating the Betti numbers of $M$ (coefficients $\mathbf{Q}$ or $\mathrm{Z}_{p}$ for $p$ prime) and those of the fixed point set of the group (see [3, p. 5]). His proof relies on the construction of a function $\varphi: M \rightarrow \mathbf{R}$ whose critical submanifolds are exactly the components of the fixed point set of the group. The function $\varphi$ constructed by Frankel has the properties of our $f$ in (1.1) except that, since it goes to $\mathbf{R}$, there is no need to consider the height functions and, in general, he does not want isolated critical points. In Corollary 2 (see p. 6) Frankel considers the case of isolated fixed points, and that corollary is a special case of (1.1); namely, the situation $N=1$. As another corollary of his main theorem Frankel obtains a proof of Bott's result (1.2) by considering the fixed point set of a maximal torus in $M$. 
We must point out that the construction of Frankel's function, which even in the case of isolated fixed points is nontrivial, provides an example of the existence of functions with the properties required in (1.1).

We now give a proof of (1.2) directly as a consequence of (1.1).

Proof of (1.2). First we notice that the hypothesis of simple connectedness can be replaced by semisimplicity, and we recall the following fact which is well known and easy to prove.

(2.1) Lemma. If $G$ is compact semisimple, a subgroup $K \subset G$ is the centralizer of a torus in $G$ if and only if there is an orbit of type $G / K$ in the adjoint representation of $G$ on its Lie algebra $g$.

Then there is an element $E \in g$ whose orbit is $G / K$. We may take $E$ in the center of $k$, the Lie subalgebra of $g$ corresponding to $K$. In $g$, as usual, we put the scalar product $\langle X, Y\rangle=-B(X, Y)$, where $B$ is the Killing form in $g$, and so $(g,\langle\rangle$,$) is a Euclidean space.$

Define now $f: M \rightarrow g$ by $f(g \cdot 0)=\operatorname{ad}(g) \cdot E \forall g \in G$, where $0=[K]$, and put on $M$ the induced metric. We have to show $f$ satisfies the conditions of (1.1). From Sard's theorem it follows that $f$ is nondegenerate and, since $f$ is an imbedding, it is well known that, for every height function $h_{v}$, the Hessian of $\left(h_{v} \circ f\right)$ is given, at a critical point $p$, by the formula

$$
\left.H\left(h_{v} \circ f\right)\right|_{p}(X, Y)=\left\langle A_{v}(X), Y\right\rangle,
$$

where $X, Y \in T_{p}(M)$ and $A_{v}$ is the shape operator of the normal vector $v$ at the point $p \in M$.

Let us recall now the following standard fact (Borel [1], Wang [7]).

(2.3) Theorem. If $K$ is the centralizer of a torus in $G$, then $G / K$ has $G$ invariant almost complex structures.

Let now $m$ be the orthogonal complement of $k$ in $g$; then, at the point $0 \in M$, we have

$$
\left.f_{*}\right|_{0}\left(T_{0}(M)\right)=[m, E]=m, \quad T_{0}(M)^{\perp}=k .
$$

Let $J$ denote a $G$-invariant almost complex structure on $M$. The $G$-invariance implies that, for each $Y \in R, X \in m$ we have, at the point $0 \in M$,

$$
\operatorname{ad}(Y) J_{0}[X, E]=J_{0} \operatorname{ad}(Y)[X, E] .
$$

Now a straightforward computation yields the expression for the shape operator at the point $0 \in M$,

$$
A_{\xi}=-(\operatorname{ad}(E))^{-1} \circ \operatorname{ad}(\xi),
$$

for every $\xi \in T_{0}(M)^{\perp}=h$.

Note. $\operatorname{ad}(E): m \rightarrow m$ is invertible and commutes with $\operatorname{ad}(\xi) \quad \forall \xi \in R$.

Now, from (2.4) and (2.5), we conclude that $A_{\xi}$ commutes with the almost complex structure at 0 . Now the $G$-invariance of $J$ and the fact that $f$ is 
isometric and equivariant yield the same conclusion at every point of $M$. With this, formula (2.2) implies that the linear Hessian has the required properties, and the conclusion of (1.2) follows.

The imbedding $f$ in the proof of (1.2) is the one considered by S. Kobayashi in [4] for compact homogeneous Kähler manifolds. Since we took $G$ semisimple after (2.1) our result yields a different proof for the simply connected case. However, as N. Kuiper points out in [5, p. 218], one can get a tight imbedding of any compact homogeneous Kähler manifold $G / K$ (other than a torus) in $\mathbf{R}^{\operatorname{dim} G}$. The example in [3, p. 7] and the situation of $S^{6} \subset \mathbf{R}^{7}$ show that (1.1) is not contained in (1.2).

\section{3}

This section contains the proof of (1.1). Let $v \in S^{N-1}$ be a vector such that the function $\varphi_{v}$ is a Morse function, and let $p$ be a critical point of $\varphi_{v}$. Let $A: T_{p}(M) \rightarrow T_{p}(M)$ be the linear Hessian at the point $p$. By condition (ii), $A$ commutes with $J_{p}$ in $T_{p}(M)$, and this in turn implies that the subspace of $T_{p}(M)$ corresponding to the negative eigenvalues is $J_{p}$-invariant. This clearly means that the index of $\varphi_{v}$ at the point $p$ is even. Since $p$ is an arbitrary critical point of $\varphi_{v}$, we have

$$
\mu\left(\varphi_{v}\right)=\sum_{i \geq 0}(-1)^{i} \mu_{i}\left(\varphi_{v}\right),
$$

and, since $\varphi_{v}$ is a Morse function, we know the equality [5, p. 210]

$$
\sum_{i \geq 0}(-1)^{i} \mu_{i}\left(\varphi_{v}\right)=\sum_{i \geq 0}(-1)^{i} \beta_{i}(M, F)=\chi(M) .
$$

Then, from (3.1) and (3.2), we obtain

$$
\mu\left(\varphi_{v}\right)=\chi(M),
$$

which clearly implies that $f$ is tight, $\beta_{i}(M, F)=0$ for $i$ odd and, since $\chi(M)$ does not depend on $F$, the even dimensional homology modules have no torsion. This concludes the proof of (1.1).

\section{ACKNOWLEDGMENTS}

We thank Gudlaugur Thorbergsson for bringing [2] to our attention. We also thank the referee for indicating the close connection between the first version of (1.1) and the paper of T. Frankel [3]. His remarks lead to the general statement of (1.1).

\section{REFERENCES}

1. A. Borel, Kählerian coset spaces of semisimple Lie groups, Proc. Nat. Acad. Sci. U.S.A. 40 (1954), 1147-1151.

2. R. Bott, An application of Morse theory to the topology of Lie groups, Bull. Soc. Math. France 84 (1956), 251-281. 
3. T. Frankel, Fixed points and torsion on Kähler manifolds, Ann. of Math. 70 (1959), 1-8.

4. S. Kobayashi, Imbeddings of homogeneous spaces with minimum total curvature, Tohoku Math. J. 19 (1967), 63-70.

5. N. H. Kuiper, Minimal total absolute curvature for immersions, Invent. Math. 10 (1970), 209-238.

6. _ Tight imbedding and maps. Submanifolds of geometric class three in $E^{N}$, Chern Symposium (Berkeley 1979), Springer-Verlag, Berlin, Heidelberg, and New York, 1980, pp. 97-145.

7. H. C. Wang, Closed manifolds with a homogeneous complex structure, Amer. J. Math. 76 (1954), 1-32.

Facultad de Matemática, Astronomia y Fisica, Universidad Nacional de Córdoba, Valparaiso y Rogelio Martinez, Ciudad Universitaria, 5000 Córdoba, Argentina 